\title{
CORPECTOMIA CERVICAL ANTERIOR E FIXAÇÃO COM PLACA: ANÁLISE RETROSPECTIVA
}

\author{
ANTERIOR CERVICAL CORPECTOMY AND PLATE FIXATION: A RETROSPECTIVE ANALYSIS
}

\author{
CORPORECTOMÍA CERVICAL ANTERIOR Y FIJACIÓN CON PLACA: \\ UN ANÁLISIS RETROSPECTIVO
}

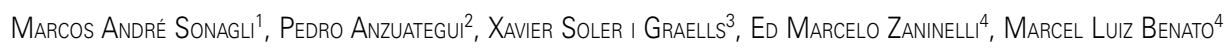

\begin{abstract}
RESUMO
Objetivo: Avaliar os resultados clínicos e radiográficos de pacientes submetidos à corpectomia e fixação com placa cervical, com seguimento de dois anos. Métodos: Análise retrospectiva de 2003 a 2009. Avaliaram-se o tipo de fratura (classificação AO), o grau de déficit neurológico (inicial e após dois anos - escala de Frankel), a taxa de complicações e a taxa de incorporação do enxerto ósseo (de acordo com radiografias dois anos depois da cirurgia). Resultados: Vinte e um pacientes foram avaliados. De acordo com a classificação AO, 14 eram grupo A, 3 B e 4 C. Ao todo, sete pacientes apresentaram déficit neurológico inicial completo (Frankel A) e permaneceram com o déficit neurológico completo após dois anos. Dos seis pacientes que apresentaram déficit neurológico inicial incompleto (Frankel B, C e D), 33\% (2 de 6) apresentaram melhora de um nível na escala de Frankel e 50\% (3 de 6) deles evoluíram para recuperação completa (Frankel E). Os oito pacientes que não apresentaram lesão neurológica inicial (Frankel E) permaneceram sem déficit neurológico após dois anos. Três complicações clínicas foram verificadas: uma fístula esofágica, uma soltura asséptica do implante e uma infecção no sítio doador de enxerto. Todos os pacientes obtiveram consolidação do enxerto ósseo. Conclusão: A corpectomia cervical no tratamento da fratura-explosão permite a recuperação neurológica nos pacientes com lesão neurológica incompleta e apresenta baixos índices de complicações.
\end{abstract}

Descritores: Coluna vertebral/cirurgia; Fusão vertebral/métodos; Procedimentos ortopédicos/instrumentação; Próteses e implantes.

\begin{abstract}
Objective: To evaluate the clinical and radiographic results of patients who underwent corpectomy and cervical plate fixation surgery with two years of follow-up. Methods: Retrospective analysis from 2003 to 2009. We evaluated the fracture type (AO classification), the degree of neurological deficit (initial and after two years - Frankel scale), the complication rate and the rate of bone graft incorporation (according to $X$-rays two years after surgery). Results: 21 patients were evaluated. According to the AO classification: 14 were in group A, 3 in $B$ and 4 in C. Overall, seven patients had initial complete neurological deficit (Frankel A) and remained with neurological complete deficit after two years of follow-up. Of the 6 patients who had incomplete initial neurological deficit (Frankel B, C and D), 33\% (2 of 6) showed an improvement on the Frankel scale level and 50\% (3 of 6) of these progressed to full recovery (Frankel E). The 8 patients without initial neurological injury (Frankel E) remained unaffected after two years. Three clinical complications were observed: an esophageal fistula, an aseptic loosening of the implant and an infection in the graft donor site. All patients had bone graft consolidation. Conclusion: Cervical corpectomy in the treatment of burst fractures allows neurological recovery in patients with incomplete neurological injury and has low complication rates.
\end{abstract}

Keywords: Spine/surgery; Spinal fusion/methods; Orthopedic. procedures/instrumentation; Prostheses and implants.

\section{RESUMEN}

Objetivo: Evaluar los resultados clínicos y radiográficos de los pacientes sometidos a corporectomía y fijación con placa cervical con dos años de seguimiento. Métodos: Análisis retrospectivo desde 2003 hasta 2009. Se evaluó el tipo de fractura (Clasificación AO), el grado de déficit neurológico (inicial y después de 2 años - escala de Frankel), la tasa de complicaciones y la tasa de incorporación del injerto óseo (de acuerdo a los rayos X de dos años de después de la cirugía). Resultados: Veintiún pacientes fueron evaluados. De acuerdo con la clasificación AO, 14 estaban en el grupo A, 3 en el B y en el C. 4 En total, siete pacientes tenían déficit neurológico inicial completo (Frankel A) y mantuvieron el déficit neurológico después de dos años. De los 6 pacientes que tenían déficit neurológico inicial incompleto (Frankel B, C y D), el 33\% (2 de 6) mostraron una mejoría en el nivel de escala de Frankel y el 50\% (3 de 6) de estas ha progresado hasta la recuperación completa (Frankel E). Los 8 pacientes sin lesión neurológica inicial (Frankel E) no se vieron afectados por déficit neurológico después de dos años. Tres complicaciones clínicas fueron observadas: una fístula, un aflojamiento aséptico del implante y una infección en la zona donante del injerto. Todos los pacientes alcanzaron una consolidación de injerto óseo. Conclusión: la corporectomía cervical en el tratamiento de las fracturas por estallido permite la recuperación neurológica de pacientes con lesión incompleta y tiene bajas tasas de complicación.

\section{Descriptores: Columna vertebral/cirugía; Fusión vertebral/métodos; Procedimientos ortopédicos/instrumentación; Prótesis e implantes.}

1. Médico Ortopedista. Especialista em Cirurgia da Coluna Vertebral pela Sociedade Brasileira de Coluna. Mestre em Clínica Cirúrgica pela UFPR.

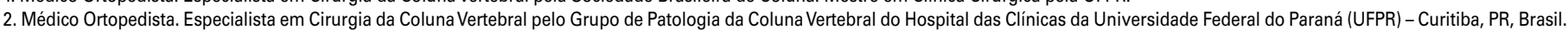

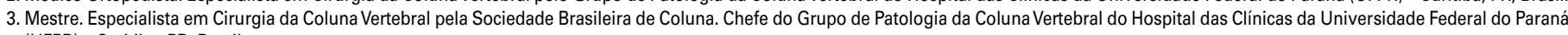
(UFPR) - Curitiba, PR, Brasil.

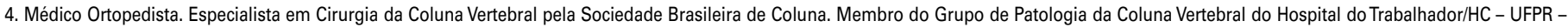
Curitiba, PR, Brasil.

Trabalho realizado no Grupo de Cirurgia da Coluna Vertebral do Hospital das Clínicas e Hospital do Trabalhador da Universidade Federal de Paraná - UFPR - Curitiba, PR, Brasil. Correspondência: Rua Guaporé, 117-D ap.402, Chapecó, SC. Brasil. CEP 89801-085. E-mail: msonagli @yahoo.com 


\section{INTRODUÇÃO}

As lesões mais frequentemente sofridas por pacientes traumatizados são as lesões esqueléticas e cranianas. As lesões esqueléticas ocorrem em $78 \%$ dos pacientes politraumatizados, sendo duas vezes maior que as lesões torácicas e quatro vezes as lesões abdominais ${ }^{1}$. O trauma da coluna vertebral é menos comum, somando $6 \%$ de todas as lesões esqueléticas. Cerca de metade destes pacientes apresentam associação com comprometimento neurológico. O dano neurológico irreversível tem profundas implicações para o paciente e para o sistema de apoio médico e social, exigindo readaptação social e provável incapacitação profissional. O fardo funcional imposto pelas lesões medulares é ampliado pelo fato da população afetada ser jovem (entre 15 e 24 anos) e ainda maior quando se considera a elevada a incidência de mortalidade (17\%) durante a hospitalização inicial dos pacientes com lesão medular ${ }^{1,2}$.

A coluna cervical está envolvida em mais da metade de todas as lesões da coluna vertebral em pacientes traumatizados. A prevalência de fratura cervical é elevada nos pacientes que apresentam déficits neurológicos focais $(19,7 \%)$ ou grave traumatismo craniano $(7,2 \%)$. Outros indicadores de lesão na coluna cervical são os mecanismos de alto impacto e a idade superior a 50 anos. O paciente com mais de 50 anos pode ter sofrido uma lesão cervical, mesmo que o mecanismo causador tenha sido de baixo impacto? .

As fraturas explosivas e as fraturas compressivas da coluna cervical se manifestam depois de ser exercida uma carga axial mecânica. Isso também foi demonstrado com auxílio de modelos matemáticos $^{3}$. O espectro das fraturas explosivas é variado. As variações podem incluir fraturas com diástase associadas a lesões da coluna posterior, pequenas fraturas em gota de lágrima e a explosão vertebral completa.

As complicações das fraturas do corpo vertebral incluem a instabilidade mecânica e neurológica. Associadas a luxações facetárias bilaterais, as fraturas explosivas da coluna cervical representam um significativo fator de risco de lesões neurológicas e da medula espinhal. A instabilidade neurológica pode ser atribuída à compressão direta da medula espinhal ou à compressão de raízes nervosas, pois as fraturas explosivas podem ocasionar a retropulsão do osso e do disco. A função neurológica inicial e evolução dependem do diâmetro do canal medular ${ }^{4}$. Por isso, um dos objetivos da cirurgia é a descompressão.

Além da instabilidade neurológica, a instabilidade mecânica é um estado em que a coluna cervical não consegue mais tolerar cargas fisiológicas normais. Infelizmente, a estabilidade mecânica não depende do grau da fratura, como nas fraturas em gota de lágrima, nas quais há lesão maior dos ligamentos devido à flexão extrema. Portanto o tratamento das fraturas explosivas inclui a descompressão neural e a restauração da estabilidade mecânica ${ }^{5}$.

A corpectomia cervical associada à fixação tem sido utilizada nos pacientes traumatizados com sucesso desde a publicação de 1988 de Cabanela e Ebersold ${ }^{6}$ que ainda utilizava placas de metacarpianos para a fixação cervical. Este autor descreveu oito pacientes tratados cirurgicamente por fratura em gota de lágrima, dos quais seis haviam fraturado $\mathrm{C} 5,1$ havia fraturado $\mathrm{C} 6$ e o outro $\mathrm{C} 7$. Deste grupo, cinco pacientes apresentavam déficit neurológico completo e três, incompleto. Após um acompanhamento médio de três anos, houve incorporação do enxerto em todos os casos, com manutenção do alinhamento sagital sem ocorrer alguma complicação relacionada aos implantes. Todos os pacientes que apresentavam déficit neurológico incompleto apresentaram algum grau de marcha independente na sua última avaliação.

Em 1993, Barros ${ }^{7}$ descreveu seus resultados cirúrgicos em 68 pacientes traumatizados e tetraparéticos tratados com corpectomia cervical. Desde grupo, quatro apresentavam fratura de C4, 24 de C5, 32 de C6 e oito de C7. Trinta pacientes sofreram lesão neurológica completa, enquanto que 38, incompleta. Do total, houve seis óbitos precoces, portanto, dos 62 pacientes incluídos na análise, cinco apresentaram alguma complicação relacionada ao procedimento. Houve cinco solturas parciais da placa/parafuso sem repercussão clínica e uma soltura total dos implantes que exigiu revisão cirúrgica. Todos os pacientes apresentaram incorporação do enxerto.

Segundo Fisher et al. $^{8}$, o tratamento cirúrgico através de corpectomia e placa cervical das fraturas explosivas em gota de lágrima é mais adequado quando comparado com o tratamento conservador. Este autor conduziu uma pesquisa na qual um grupo de 45 pacientes vítimas de fratura em gota de lágrima foram divididos em tratamento conservador com halo-colete (21 pacientes) e tratamento cirúrgico com corpectomia (24 pacientes). No grupo tratado conservadoramente foi observado cifose residual de $11,4^{\circ}$ em média, em comparação com o outro grupo no qual a média foi $3,5^{\circ}$. Houve falha do tratamento conservador em cinco pacientes e, portanto, na evolução foram submetidos ao tratamento cirúrgico. A avaliação final da qualidade de vida, através do questionário MOS36, no entanto, foi semelhante para ambos os grupos.

A corpectomia cervical tornou-se um procedimento comum dentro da cirurgia de coluna e é indicado não somente no trauma, mas também para descompressão da medula em numerosas doenças degenerativas, neoplásicas e infecciosas. Conforme descreve Özgen et al. ${ }^{9}$ em seu estudo retrospectivo de 72 pacientes submetidos à corpectomia cervical, no qual $36 \%$ eram portadores de doença degenerativa, 25\% foram vítimas de trauma, 15\% apresentavam algum tipo de neoplasia, 14\% infecção e 10\% ossificação do ligamento longitudinal posterior. Nesta série, 52\% dos casos operados compreendiam apenas um nível. Özgen et al. ${ }^{9}$ encontraram 31 complicações em 15 pacientes: 12 complicações cirúrgicas, cinco relacionadas ao enxerto, sete relacionadas ao implante e sete complicações clínicas. Este autor relata 93\% de incorporação do enxerto.

Eleraky et al. ${ }^{10}$ conduziram estudo com 185 pacientes submetidos à corpectomia cervical entre 1987 e 1998. Destes, 81 por espondilose degenerativa, 16 por ossificação do ligamento longitudinal posterior, 39 por trauma, 10 por tumor, 31 por correção de cifose pós-operatória e oito por infecção. Enxerto da crista ilíaca foi utilizado em 141 casos e da fíbula nos demais pacientes. Não houve seqüela neurológica. Rouquidão, disfagia e dor no local doador do enxerto foram tratadas com sucesso. Houve 98,8\% de índice de incorporação do enxerto.

Boakye et al. ${ }^{11}$ realizaram estudo multicêntrico baseando-se em banco de dados americano com 1560 pacientes submetidos à corpectomia cervical entre 1997 e 2006 por doença não exclusivamente traumática. Este autor encontrou mortalidade hospitalar de 1,6\% e uma taxa de complicações de 18,4\% com permanência hospitalar média de seis dias. Os maiores fatores preditivos de complicações foram: idade acima de 80 anos, história de diabetes tipo I e avaliação pré-anestésica com ASA maior ou igual a três. Neste estudo, os pacientes que foram submetidos à corpectomia em três ou mais níveis tiveram uma taxa de re-operação de 18\% e de complicação relacionada ao enxerto/implante de 5,4\%, contra $6,2 \%$ e $1,8 \%$, respectivamente, daqueles que foram submetidos à corpectomia em apenas um nível.

Outros autores relataram possíveis complicações raras nos pacientes submetidos à corpectomia cervical. Yen et al. ${ }^{12}$ descreveram dois casos de fratura do implante decorrente de pseudoartrose em pacientes submetidos a procedimentos em múltiplos níveis. Wang et al. ${ }^{13}$ descreveu 16 casos de migração do enxerto em 249 pacientes submetidos à corpectomia cervical por doença de base não traumática. Ele refere ser fator de risco para migração do enxerto a cirurgia executada em múltiplos níveis e quando a fixação termina em C7. Epstein et al. ${ }^{14}$ descreveram quatro casos em 56 operados de fratura tardia do enxerto tendo sido diagnosticada por tomografia computadorizada. A maioria dos pacientes apresentava dor cervical crônica, vários meses após o procedimento. Joanes e Belinchon ${ }^{15}$ descreveram um caso de divertículo faringoesofágico 
decorrente de corpectomia cervical, sendo diagnosticado após três anos do procedimento. Segundo este autor, o tecido cicatricial decorrente do mau posicionamento da placa provocou tração às vísceras que evoluíram com divertículo. Este paciente foi submetido a tratamento cirúrgico do divertículo com remoção do implante, apresentando cura.

O objetivo deste estudo foi avaliar os resultados clínicos e radiográficos de pacientes submetidos à corpectomia e fixação com placa cervical com seguimento mínimo de dois anos.

\section{MATERIAIS E MÉTODOS}

\section{Análise dos pacientes}

Entre 2003 e 2009, 26 pacientes com fraturas cervicais foram tratados com corpectomia, enxertia de crista ilíaca e fixação anterior com placa e parafusos (25 homens e uma mulher). Do total, dois pacientes apresentaram fratura em duas vértebras adjacentes e um paciente apresentou fratura em três vértebras adjacentes, totalizando 30 fraturas. Todos os pacientes apresentaram fratura do corpo vertebral associada ou não à lesão ligamentar posterior.

Foi utilizada a classificação AO para agrupamento dos pacientes. A classificação de Frankel foi empregada para graduar a lesão neurológica no atendimento inicial e sua evolução.

\section{Técnica utilizada}

O acesso anterior convencional foi empregado para a realização da corpectomia com auxílio de intensificador de imagens ${ }^{16}$. As laterais dos corpos vertebrais foram poupadas e foi realizada discectomia do nível acima e abaixo da fratura, assim como curetagem das respectivas placas terminais. O enxerto de crista ilíaca tricortical com dimensões apropriadas foi inserido no local resultante da excisão do corpo vertebral e dos discos intervertebrais. Uma placa cervical foi fixada aos corpos vertebrais adjacentes e ao enxerto, totalizando seis parafusos. O intensificador de imagens foi utilizado para determinar o comprimento dos parafusos para evitar que se ultrapassasse a cortical posterior dos corpos vertebrais.

\section{Acompanhamento pós-operatório}

Os pacientes utilizaram colar cervical tipo Philadelphia nos pós-operatório até haver sinais de incorporação do enxerto. Foi permitido ortostatismo ou sentar no leito assim que possível, o que ocorria normalmente no $3^{\circ}$ pós-operatório. Foram realizadas consultas de revisão ambulatorial para avaliação clínica e radiográfica. Em sua última avaliação ambulatorial os pacientes foram submetidos a radiografias em AP e perfil dinâmico para verificar a incorporação do enxerto e a fixação da placa.

\section{RESULTADOS}

Do total de 26 pacientes, como mecanismo do trauma foi notado: oito acidentes automobilísticos, sete quedas de nível, cinco mergulhos em água rasa, três choques contra objeto e três acidente com arma de fogo. A média de idade foi 29 anos (14-57) com seguimento mínimo de dois anos (25 - 70 meses). Houve cinco óbitos precoces não relacionados com a técnica cirúrgica. Portanto, 21 pacientes foram avaliados após dois anos de seguimento.

De acordo com a classificação AO para as fraturas cervicais, do total de 21 pacientes, 14 eram do grupo A (três do subgrupo A2 e 11 do subgrupo A3), cinco do grupo B (todas do subgrupo B2) e dois do grupo C (um do subgrupo $\mathrm{C} 1$ e um do subgrupo $\mathrm{C} 3$ ).

De acordo com a classificação de Frankel, 69\% dos pacientes apresentaram algum grau de lesão neurológica: seis com déficit incompleto, 12 com lesão neurológica completa e oito pacientes sem lesão neurológica.

Ao correlacionar o grau de lesão neurológica com a classificação da fratura observa-se que todos os dois pacientes agrupados como $\mathrm{AO}$ tipo $\mathrm{C}$ apresentavam déficit neurológico, sendo um com lesão incompleta e um com lesão completa. Ao passo que dos cinco pacientes classificados dentro do tipo B pela classificação da $A O$, três não sofreram lesão neurológica e dois apresentavam lesão incompleta. Quando analisamos os 17 pacientes que sofreram fratura do tipo $\mathrm{A}$, seis tinham dano neurológico completo, dois com dano incompleto e cinco sem algum dano (Tabela 1).

Todos os pacientes com lesão neurológica incompleta apresentaram algum grau de deambulação independente em sua última avaliação, no entanto, os pacientes com lesão completa obtiveram pouca ou nenhuma melhora sensitiva ou motora. Todos os sete pacientes que deram entrada com lesão neurológica completa permaneceram com o mesmo grau de déficit em sua última avaliação, enquanto que houve melhora de um ou mais níveis na escala de Frankel em 83\% (cinco de seis) dos pacientes com lesão incompleta, graduados de Frankel B a D, sendo que 50\% (três de seis) destes evoluíram com recuperação completa (Frankel E). Não houve deterioração neurológica naqueles pacientes que não apresentavam lesão na admissão (Tabela 2).

Houve incorporação do enxerto de crista ilíaca em todos os pacientes analisados (Figuras 1 e 2).

Tabela 1. Correlação entre os tipos de fratura pela Classificação AO e a Escala de Frankel (neurológica) na admissão.

\begin{tabular}{c|c|c|c|c|c}
\hline Classificação AO & \multicolumn{5}{|c}{ Escala de Frankel } \\
\hline & A & B & C & D & E \\
\hline A & 6 & 2 & 0 & 0 & 5 \\
\hline B & 0 & 1 & 1 & 0 & 3 \\
\hline C & 1 & 0 & 0 & 1 & 0 \\
\hline
\end{tabular}

Tabela 2. Análise da evolução do déficit neurológico após 2 anos de follow-up.

\begin{tabular}{c|c|c|c|c|c}
\hline Admissão & \multicolumn{5}{|c}{ Evolução (após 2 anos) } \\
\hline Frankel & A & B & C & D & E \\
\hline A & 7 & & & & \\
\hline B & & & 1 & 1 & 2 \\
\hline C & & & 1 & & \\
\hline D & & & & & 1 \\
\hline E & & & & & 8 \\
\hline
\end{tabular}
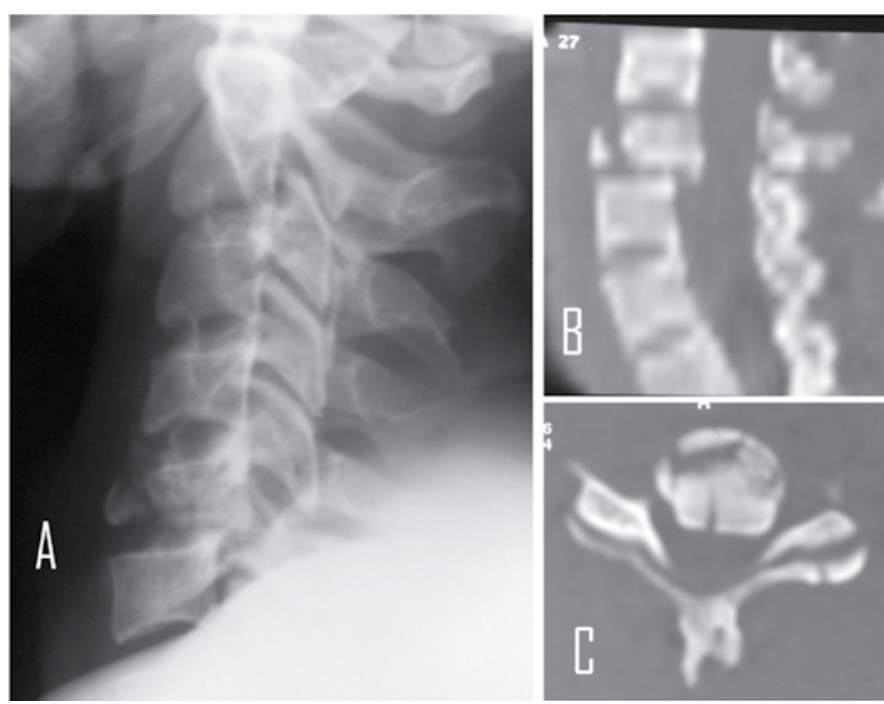

Figura 1. Paciente de 22 anos vítima de acidente de trabalho sofreu choque contra objeto dando entrada no pronto-socorro com lesão neurológica incompleta (Frankel B) e fratura-explosão de C5 (imagem A), com fragmento retropulsado para o interior do canal medular (imagens B e C). 


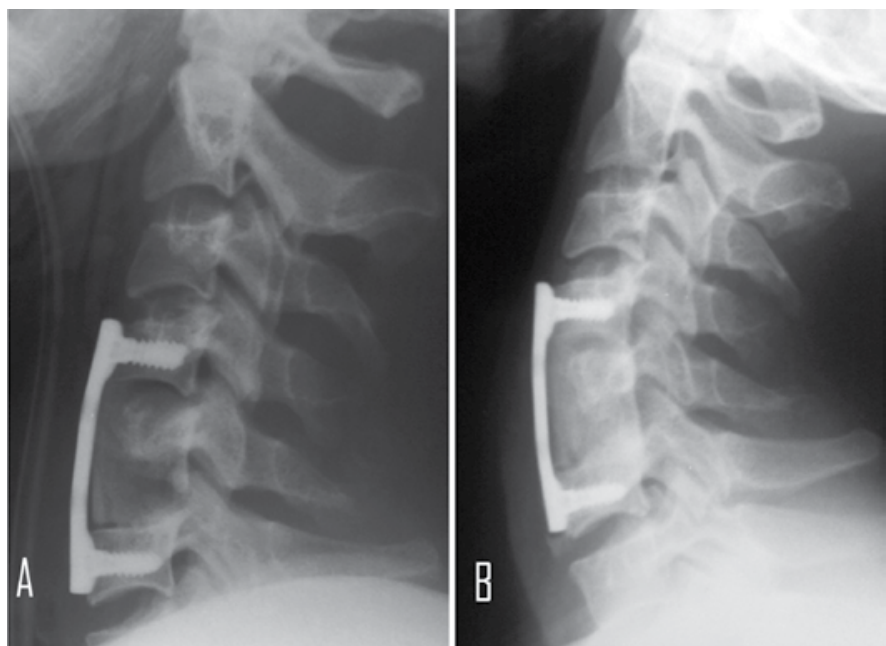

Figura 2. Incorporação do Enxerto: O mesmo paciente da Figura 1 em (A) pós-operatório imediato de corpectomia de C5, descompressão do canal medular por via anterior, enxerto estrutural de crista ilíaca e fixação com placa cervical. Com dois anos e meio (B) de pós-operatório houve recuperação completa da lesão neurológica e incorporação total do enxerto.

Houveram três complicações relacionadas ao procedimento: uma soltura asséptica do implante, uma fístula esofágica e uma infecção do sítio doador de enxerto. Foi removido o material de síntese do paciente com soltura e daquele com a fístula. Foi realizada limpeza cirúrgica associada à antibioticoterapia endovenosa para o paciente com infecção no local doador. Não houve complicação relacionada ao enxerto.

\section{DISCUSSÃO}

O acesso cervical anterior tem sido utilizado com sucesso desde os relatos de Smith e Robinson ${ }^{16}$. A possibilidade de descompressão direta do canal medular torna este acesso particularmente útil para os pacientes que sofreram fraturas explosivas da coluna cervical.

Com o objetivo de evitar cifose residual e migração do enxerto, é consenso que se associe fixação com placa cervical anterior, não havendo mais espaço para enxertia isolada ${ }^{6}$. Quando existe lesão ligamentar posterior ou o trauma sofrido causou forças torcionais, pode ser necessário associação de fixação posterior com placas ou amarria, o que infelizmente não é desejado por agregar morbidade ao tratamento.

Cabanela e Ebersold ${ }^{6}$ utilizaram em sua pesquisa inicial halo-colete no pós-operatório com o propósito de evitar soltura do implante e migração do enxerto. No entanto, em sua série, Cabanela e Ebersold ${ }^{6}$ utilizaram placas metacarpais como implante. Com a melhoria tecnológica, os novos implantes cervicais permitem fixação estável que dispensa o halo-colete até a incorporação do enxerto.

Há discussão sobre qual seria o melhor método de tratamento para as fraturas explosivas e em gota de lágrima, classificadas como flexão-compressão de Allen et al. ${ }^{17}$ no estágio 3, 4 e 5. Fisher et $\mathrm{al}^{8}$ realizaram estudo no qual dividiu 45 pacientes que apresentavam estas fraturas em dois grupos: tratamento conservador com halo-colete e tratamento cirúrgico com corpectomia, enxerto estrutural e placa cervical anterior. Os pacientes submetidos ao tratamento cirúrgico obtiveram cifose média residual na última avaliação de $3,5^{\circ}$ ao passo que o grupo tratado conservadoramente obteve $11,4^{\circ}(p<0,001)$. Houve cinco falhas do tratamento conservador necessitando de intervenção cirúrgica, dois dos quais apresentaram piora neurológica. Fisher concluiu que o tratamento cirúrgico é um método seguro e eficaz para a recuperação e a manutenção do alinhamento cervical sagital, atingindo $100 \%$ de união do enxerto com poucas complicações relatadas, ao passo que o tratamento conservador apresenta altas taxas de complicações como infecção no trajeto dos pinos, possibilidade de falha, perda da redução, levando à cifose residual e dor crônica.

Koivikko et al. ${ }^{18}$ conduziram estudo com 69 pacientes divididos em dois grupos: tratamento conservador com tração/halo-colete e tratamento cirúrgico com corpectomia descompressiva/placa cervical anterior. Ele relata que apenas quatro de 18 pacientes com algum dano neurológico (Frankel $A$ até $D$ ) que foram tratados conservadoramente apresentaram pelo menos um grau de melhora na escala de Frankel, em comparação com 13 de 23 daqueles tratados cirurgicamente $(p=0,027)$. Dentre todos os 17 pacientes que recuperaram pelo menos um grau na escala de Frankel foi significativa a redução do grau de deslocamento dos fragmentos para o interior do canal medular comparando com aqueles que não obtiveram melhora neurológica $(7,2 \%$ contra $18,3 \%, p=0,0006$ ). Ele concluiu que a melhora neurológica no grupo tratado cirurgicamente foi devido à descompressão do canal medular porque comparando o deslocamento dos fragmentos entre os dois grupos em sua última avaliação, os pacientes que foram submetidos à cirurgia apresentavam 7,4\% de invasão contra $21,5 \%$ daqueles tratados conservadoramente $(p=0.0001)$. Koivikko et al. ${ }^{18}$ observaram que o alinhamento sagital dos pacientes tratados com cirurgia foi de $2,2^{\circ}$ de lordose na última avaliação, em comparação com $12,6^{\circ}$ de cifose naqueles tratados conservadoramente $(p=00003)$.

Barros $^{7}$ relata que a recuperação neurológica dos pacientes que apresentavam na admissão lesão completa é limitada, evoluindo de 12 para 23 pontos no escore motor da ASIA (American Spine Injury Association), ao passo que a evolução daqueles pacientes com lesão incompleta é muito superior (de 30 para 72 pontos). No presente estudo foi utilizada a classificação de Frankel para graduar a lesão neurológica, que apesar de simples é amplamente utilizada clinicamente. Foi observado resultado semelhante ao de Barros, visto que nenhum paciente que na admissão foi graduado como Frankel A evoluiu com melhora nesta escala. Considerando apenas os pacientes com lesão neurológica incompleta (Frankel B a D), o presente estudo constatou $83 \%$ de melhora de pelo menos um grau na escala de Frankel, enquanto que Koivikko et al. ${ }^{18}$ relataram $81 \%$ (nove de 11 pacientes).

A superioridade dos resultados do tratamento cirúrgico esbarra nas possíveis complicações relacionadas ao procedimento, ao implante e ao enxerto. No entanto, tanto o presente estudo como os dados da literatura sustentam que estas taxas de complicações são aceitáveis. Cabanela e Ebersold ${ }^{6}$ negaram complicações em sua série inicial de oito pacientes. Barros ${ }^{7}$ relata seis complicações relacionadas aos implantes em sua série de 68 pacientes, sendo necessário re-intervenção em um caso. Koivikko et al. ${ }^{18}$ necessitaram re-intervir cirurgicamente em 2 de 35 pacientes devido à soltura e mal posicionamento de parafusos. Fisher et al. ${ }^{8}$ negaram maiores complicações em seu estudo com 21 pacientes operados. Ozgen et al. ${ }^{9}$ relataram em sua série de 72 casos, dentre os quais, havia pacientes não-traumatizados, 15\% de complicações cirúrgicas, 4,2\% de complicações relacionadas ao enxerto e 9,7\% de complicações relacionadas aos implantes. No entanto, Ozgen et al. ${ }^{9}$ necessitaram re-operar apenas dois destes pacientes.

No presente estudo foram observadas três complicações maiores. Houve uma lesão esofágica com evolução para fístula que necessitou intervenção conjunta com a equipe de cirurgia geral após quatro meses do trauma. Foi notado no per-operatório que havia incorporação completa do enxerto e, portanto os implantes foram removidos. No outro caso foi notado soltura parcial asséptica da placa cervical com cinco meses de pós-operatório, manifestando-se clinicamente com cervicalgia crônica. Foi optado por remoção do implante com resolução do quadro. A terceira complicação foi um caso de infecção profunda com ausência de osteomielite no local doador do enxerto de crista ilíaca. Após limpeza cirúrgica e antibióticos o quadro foi completamente resolvido. 
O presente estudo retrata bons resultados no tratamento de um tipo de fratura que pode causar conseqüências desastrosas na vida de pacientes geralmente jovens e em idade produtiva. $\mathrm{Na}$ presença de traumatismo raquimedular o fardo social e familiar é ainda mais pronunciado. Os acidentes automobilísticos responderam pela maioria dos casos nesta série, tomando o lugar de quedas de altura e mergulho em água rasa. $O$ tratamento destas fraturas tem seguido uma tendência mais agressiva e intervencionista, reabilitando mais precocemente estes jovens à sociedade, no entanto, investimento público de prevenção com políticas educacionais de trânsito poderiam evitar grande parte das colisões, poupando a vida ou evitando sequelas permanentes nestes pacientes.

\section{CONCLUSÃO}

A corpectomia cervical no tratamento da fratura-explosão permite a recuperação neurológica nos pacientes com lesão neurológica incompleta e apresenta baixos índices de complicações.

\section{REFERÊNCIAS}

1. Sohail K.Mirza. Principles of Spine Trauma Care. Chapter 37. In: Robert W, Heckman JD editors. Paginas: 1402-3. Rockwood and Green's Fractures in adults, Lippincott Williams \&Wilkins; 6th edition. Pages 1402-3. 881, USA.

2. Vaccaro AR, Betz RR, Zeidman SM. Cirurgia da coluna: princípios e prática. Rio de Janeiro: Dilivros; 2007

3. Bozic KJ, Keyak JH, Skinner HB, Bueff HU, Bradford DS. Three-dimensional finite element modeling of a cervical vertebra: an investigation of burst fracture mechanism. J Spinal Disord. 1994;7(2):102-10.

4. Sapkas G, Korres D, Babis GC, Efstathiou P, Papaioannou N, Antoniadis A, et al. Cor relation of spinal canal post-traumatic encroachment and neurological deficit in burst fractures of the lower cervical spine (C3-7). Eur Spine J. 1995;4(1):39-44.

5. Aebi M, Zuber K, Marchesi D. Treatment of cervical spine injuries with anterior plating. Indications, techniques, and results. Spine (Phila Pa 1976). 1991;16(Suppl 3):S38-45.

6. Cabanela ME, Ebersold MJ. Anterior plate stabilization for bursting teardrop fractures of the cervical spine. Spine (Phila Pa 1976). 1988;13(8):888-91.

7. Barros T. Corpectomy and anterior plating in cervical spine fractures with tetraplegia. Rev Paul Med. 1993;111(3):2.

8. Fisher CG, Dvorak MF, Leith J, Wing PC. Comparison of outcomes for unstable lower cervical flexion teardrop fractures managed with halo thoracic vest versus anterior corpectomy and plating. Spine (Phila Pa 1976). 2002;27(2):160-6.

9. Ozgen S, Naderi S, Ozek MM, Pamir MN. A retrospective review of cervical corpectomy: indications, complications and outcome. Acta Neurochir (Wien). 2004:146(10):1099-105. 10. Eleraky MA, Llanos C, Sonntag VK. Cervical corpectomy: report of 185 cases and review of the literature. J Neurosurg. 1999;90(Suppl 1):35-41.

11. Boakye M, Patil CG, Ho C, Lad SP. Cervical corpectomy: complications and outcomes. Neurosurgery. 2008;63(4 Suppl 2):295-301.

12. Yen $\mathrm{CP}$, Hwang TY, Wang CJ, Howng SL. Fracture of anterior cervical plate implant--report of two cases. Acta Neurochir (Wien). 2005;147(6):665-7.

13. Wang JC, Hart RA, Emery SE, Bohlman HH. Graft migration or displacement after multilevel cervical corpectomy and strut grafting. Spine (Phila Pa 1976). 2003:28(10):1016-21.

14. Epstein NE, Dickerman RD. Delayed iliac crest autograft fractures following plated singlelevel anterior cervical corpectomy with fusion. J Spinal Disord Tech. 2002;15(5):420-4.

15. Joanes V, Belinchon J. Pharyngoesophageal diverticulum following cervical corpectomy and plating. Case report. J Neurosurg Spine.2008;9(3):258-60.

16. Smith GW, Robinson RA. The treatment of certain cervical-spine disorders by anterior removal of the intervertebral disc and interbody fusion. J Bone Joint Surg Am. 1958;40(3):607-24.

17. Allen $B L J$ r, Ferguson $R L$, Lehmann TR, O'Brien RP. A mechanistic classification of closed, indirect fractures and dislocations of the lower cervical spine. Spine (Phila Pa 1976). $1982 ; 7(1): 1-27$.

18. Koivikko MP, Myllynen P, Karjalainen M, Vornanen M, Santavirta S. Conservative and operative treatment in cervical burst fractures. Arch Orthop Trauma Surg. 2000;120(7-8):448-51. 